\title{
1. A Monte-Carlo simulation of the equilibrium beam polarization in ultra-high energy electron(positron) storage rings
} ?

\author{
Zhe Duan ${ }^{\mathrm{a}, \mathrm{b}, *}$, Mei Bai ${ }^{\mathrm{c}}$, Desmond P. Barber ${ }^{\mathrm{d}}$, Qing Qin ${ }^{\mathrm{a}}$ \\ Key Laboratory of Particle Acceleration Physics and Technology, Institute of High Energy \\ Physics, Chinese Academy of Sciences, 100049 Beijing, China \\ ${ }^{b}$ University of Chinese Academy of Sciences, 100049 Beijing, China \\ ${ }^{c}$ Forschungszentrum Jülich GmbH, 52428 Jülich, Germany \\ ${ }^{d}$ Deutsches Elektronen-Synchrotron, DESY, 22607 Hamburg, Germany
}

10 Abstract

With the recently emerging global interest in building a next generation of circular electron-positron colliders to study the properties of the Higgs boson, and other important topics in particle physics at ultra-high beam energies, it is also important to pursue the possibility of implementing polarized beams at this energy scale. It is therefore necessary to set up simulation tools to evaluate the beam polarization at these ultra-high beam energies. In this paper, a MonteCarlo simulation of the equilibrium beam polarization based on the Polymorphic Tracking Code(PTC) [1] is described. The simulations are for a model storage ring with parameters similar to those of proposed circular colliders in this energy range, and they are compared with the suggestion [2] that there are different regimes for the spin dynamics underlying the polarization of a beam in the presence of synchrotron radiation at ultra-high beam energies. In particular, it has been suggested that the so-called "correlated" crossing of spin resonances during synchrotron oscillations at current energies, evolves into "uncorrelated" crossing of spin resonances at ultra-high energies.

11 Keywords: Monte-Carlo simulation, Equilibrium beam polarization,

12 Electron(positron) storage rings

${ }_{13} \quad P A C S: 29.27 . \mathrm{Hj}, 29.20 . \mathrm{db}$

* Corresponding author. Tel.: +86 1088235504

Email address: zhe.duan@ihep.ac.cn (Zhe Duan )

Preprint submitted to Nuclear Instruments and Methods in Physics Research Section A: Accelerators, Spectrometers, Detectors and

(C) 2015. This manuscript version is made available under the Elsevier user license http://www.elsevier.com/open-access/userlicense/1.0/ 


\section{Introduction}

With the discovery of the Higgs boson by the ATLAS and CMS experiments at CERN's Large Hadron Collider(LHC), it becomes natural to measure its properties as precisely as possible, at the LHC or future electron-positron colliders. Besides the International Linear Collider, there is an alternative possibility, namely to build a circular electron-positron collider, with the merit of a higher luminosity-to-cost ratio, and the potential that it be upgraded to a proton-proton collider later. Currently, there are two major design studies of such a circular electron positron collider in the world, the CEPC [3] and the FCC-ee(TLEP) [4]. These designs open the possibility of precision measurements at the $\mathrm{Z}$ pole, at the WW threshold, at the HZ cross section maximum, and even at the $t \bar{t}$ threshold, with an unprecedented accuracy. Then it is natural to pursue the possibility of implementing polarized electron and positron beams at these ultra-high beam energies, for precision beam energy calibration with transverse beam polarization, or colliding-beam experiments with longitudinal beam polarization.

The motion of the spin expectation value (the "spin") $\vec{S}$ of a relativistic charged particle traveling in electromagnetic fields is described by the ThomasBMT equation $[5,6]$,

$$
\begin{aligned}
\frac{d \vec{S}}{d \theta} & =\vec{\Omega} \times \vec{S} \\
\vec{\Omega}(z, \theta) & =\vec{\Omega}_{0}(\theta)+\vec{\omega}(z, \theta),
\end{aligned}
$$

where $\theta$ is the azimuthal angle, $\vec{\Omega}_{0}$ is due to the fields on the closed orbit, and $\vec{\omega}$ is due to the fields at the beam coordinates $\vec{z}=\left(x, p_{x}, y, p_{y}, z, \delta\right)$ with respect to the closed orbit, where $x, y$ and $z$ represent the horizontal, vertical and longitudinal displacements, $p_{x}$ and $p_{y}$ are horizontal and vertical canonical momenta, and $\delta=\Delta E / E_{0}$ is the relative energy deviation. As we shall see, it is often necessary to describe spin motion with the help of a unit vector field $\hat{n}(z, \theta)$ (the "invariant spin field", or ISF for short) [7]. This satisfies the Thomas-BMT equation along particle trajectories and it is periodic: $\hat{n}(\vec{z} ; \theta)=\hat{n}(\vec{z} ; \theta+2 \pi)$. On 
the closed orbit $\hat{n}$ is denoted by $\hat{n}_{0}$. In the rings treated here, $\hat{n}_{0}$ is nominally vertical in the arcs. The rate of precession of spins around $\hat{n}$ is characterized by the amplitude dependent spin tune $\nu_{s}$ [7]. This reduces to the closed-orbit spin tune, $\nu_{0}$, on the closed orbit. In a perfectly aligned planar ring, $\nu_{0}=a \gamma_{0}$, where $a=0.00115965219$ for electrons(positrons), and $\gamma_{0}$ is the relativistic factor for the design energy. At orbital tunes for which constituent terms in the perturbation $\vec{\omega}$ stay coherent over long periods with the basic spin precession, $\hat{n}(\vec{z} ; \theta)$ can deviate strongly from $\hat{n}_{0}(\theta)$. This phenomenon is called spin-orbit resonance (which will simply call "spin resonance" or "resonance" here) and the condition is

$$
\nu_{s}=k+k_{x} \nu_{x}+k_{y} \nu_{y}+k_{z} \nu_{z}, \quad k, k_{x}, k_{y}, k_{z} \in \mathbb{Z} .
$$

where $\nu_{x}, \nu_{y}$ and $\nu_{z}$ are the orbital tunes. We expect that if, for some reason, a particle suffers large uncorrelated jumps in its orbital phases, the coherence is lost and the resonances can be suppressed.

In storage rings, electron and positron beams become spontaneously polarized due to the spin-flip synchrotron radiation, this phenomenon is called the Sokolov-Ternov effect [8]. On the other hand, the stochastic (synchrotronradiation) photon emissions modify the trajectories and thus the $\vec{\omega}$ along the trajectories. Then spin diffusion and thus depolarization can occur [9]. The polarization is therefore a balance between the Sokolov-Ternov effect and the radiative depolarization effect. In the seminal paper of Derbenev and Kondratenko [10], they derived the renowned formula of the equilibrium beam polarization in an electron(positron) storage ring, which is along the direction of $\hat{n}$ at each point in phase space, and with a magnitude of

$$
\begin{aligned}
P_{\mathrm{dk}} & =-\frac{8}{5 \sqrt{3}} \frac{\alpha_{-}}{\alpha_{+}} \\
\alpha_{-} & =\oint \mathrm{d} \theta\left\langle\frac{1}{|\rho|^{3}} \hat{b} \cdot\left(\hat{n}-\frac{\partial \hat{n}}{\partial \delta}\right)\right\rangle \\
\alpha_{+} & =\oint \mathrm{d} \theta\left\langle\frac{1}{|\rho|^{3}}\left[1-\frac{2}{9}(\hat{n} \cdot \hat{\beta})^{2}+\frac{11}{18}\left(\frac{\partial \hat{n}}{\partial \delta}\right)^{2}\right]\right\rangle,
\end{aligned}
$$


where $\partial \hat{n} / \partial \delta$ is the so-called spin-orbit coupling function, which quantifies the depolarization, and which can be very large near spin resonances. $\hat{\beta}$ is a unit vector along the direction of particle motion, $\dot{\hat{\beta}}$, the slope of $\hat{\beta}$ is therefore along the direction of acceleration, and $\hat{b}=\hat{\beta} \times \dot{\hat{\beta}} /|\dot{\hat{\beta}}|$. The brackets \langle\rangle denote an average over phase space at azimuth $\theta$. The term with $\partial \hat{n} / \partial \delta$ in $\alpha_{-}$accounts for the so-called kinetic polarization effect. In rings where $\hat{n}_{0}$ is nominally vertical in the arcs this term is negligible. Following the definitions used in Ref. [2], the polarization build-up rate is

$$
\lambda_{\mathrm{dk}}=\lambda_{p}+\lambda_{d}^{0},
$$

where $\lambda_{p}$ and $\lambda_{d}^{0}$ are the rates of the Sokolov-Ternov effect and the depolarization effect, respectively,

$$
\begin{aligned}
& \lambda_{p}=\frac{5 \sqrt{3}}{8} \frac{r_{e} \gamma^{5} \hbar}{m_{e}} \frac{C}{2 \pi c} \oint \mathrm{d} \theta\left\langle\frac{1-\frac{2}{9}(\hat{n} \cdot \hat{\beta})^{2}}{|\rho|^{3}}\right\rangle \\
& \lambda_{d}^{0}=\frac{5 \sqrt{3}}{8} \frac{r_{e} \gamma^{5} \hbar}{m_{e}} \frac{C}{2 \pi c} \oint \mathrm{d} \theta\left\langle\frac{\frac{11}{18}\left(\frac{\partial \hat{n}}{\partial \delta}\right)^{2}}{|\rho|^{3}}\right\rangle,
\end{aligned}
$$

where $r_{e}$ and $m_{e}$ are the classical radius and mass of electron, $\hbar$ is the Planck constant, $c$ is the speed of light, and $C$ is the circumference of the storage ring. Note that $\lambda_{p}$ and $\lambda_{d}^{0}$ are dimensionaless, but that one can also define the characteristic time of the Sokolov-Ternov effect $\tau_{p}=C /\left(2 \pi c \lambda_{p}\right)$ and of the depolarization effect $\tau_{d}^{0}=C /\left(2 \pi c \lambda_{d}^{0}\right)$, which can be compared with the beam lifetime and aid the analysis of beam polarization. For later use we also need

$$
\tilde{\lambda}_{p}=\frac{5 \sqrt{3}}{8} \frac{r_{e} \gamma^{5} \hbar}{m_{e}} \frac{C}{2 \pi c} \oint \mathrm{d} \theta \frac{1}{|\rho|^{3}},
$$

but since the term with $\frac{2}{9}(\hat{n} \cdot \hat{\beta})^{2}$ makes a negligible contribution for the rings considered here, $\tilde{\lambda}_{p} \approx \lambda_{p}$.

A prerequisite for estimating the attainable beam polarization in an electron(positron) storage ring is a detailed knowledge of the contributions to the depolarization. For that a simulation code must be established and that code can then serve as a guide for the design and optimization of the ring. Two classes of methods [11] have been developed for evaluating the equilibrium polarization in electron storage rings. 
One class computes $\hat{n}$ and $\partial \hat{n} / \partial \delta$ around the ring and applies Eq. 3 to obtain the equilibrium beam polarization. Various codes of this class are listed in [11], and they differ in the degree of linearization of the spin and orbit motion, as well as being perturbative or non-perturbative. The codes that handle linearized spin and orbit motion, for example SLIM [12], only describe the firstorder spin resonances, namely those with $\nu_{0}=k+k_{x} \nu_{x}+k_{y} \nu_{y}+k_{z} \nu_{z}$ for which, $\left|k_{x}\right|+\left|k_{y}\right|+\left|k_{z}\right|=1$, while higher-order spin resonances, namely those with $\left|k_{x}\right|+\left|k_{y}\right|+\left|k_{z}\right|>1$ are not taken into account. Note that higher-order spin resonances stem from the three dimensional nature of spin motion and that they can be driven by linear orbital motion. This is ultimately due to the fact that successive spin rotations around different axes do not commute. This is illustrated in the SMILE formalism [13] which evaluates the equilibrium polarization of Eq. 3 using a perturbative calculation of $\hat{n}$ and $\partial \hat{n} / \partial \delta$ in power series of the orbital amplitudes. The SMILE formalism also illustrates how to identify the sources of resonances and, importantly, how to classify them. Note also that for perturbative calculations it is $\nu_{0}$ that appears in the expression for a resonance, not $\nu_{s}$. The normal form analysis in PTC can also be used to compute $\hat{n}$ on particle trajectories perturbatively [14]. However, these perturbative approaches have convergence problems for the calculation of synchrotron sideband resonances for high beam energies [15].

Synchrotron sideband resonances are those resulting from the modulation of the rate of spin precession around $\hat{n}_{0}$ due to the energy oscillations inherent in synchrotron oscillations. For example, for first-order parent $\nu_{x}$ or $\nu_{y}$ resonances the sideband resonances satisfy the condition $\nu_{0}=k+k_{x} \nu_{x}+k_{y} \nu_{y}+k_{z} \nu_{z}$ for which, $\left|k_{x}\right|+\left|k_{y}\right|=1$ with $k_{z} \neq 0$. The occurrence of extra resonances is easy to understand when it is recalled that the modulation of a base frequency always introduces sidebands into the frequency spectrum. The modulation of the rate of spin precession around $\hat{n}_{0}$ due to energy oscillations mostly occurs in the arcs where $\hat{n}_{0}$ is nominally vertical and where particles experience a contribution to their horizontal motion in the quadrupoles due horizontal dispersion and energy oscillations. A modulation also occurs due to horizontal betatron motion in arc 
quadrupoles. However, betatron tunes are large so that the average modulation over one turn is small and then the corresponding sidebands are insignificant. In contrast, synchrotron tunes are very small so that the average modulation cannot be ignored. In any case we focus here on synchrotron motion because it is this motion that is in first instance effected by photon emission.

SODOM [16] treats linearized orbit motion and three-dimensional spin motion. The field $\hat{n}$ is computed non-perturbatively and a first-order difference of $\hat{n}$ at several nearby phase space points is used to compute $\partial \hat{n} / \partial \delta$. In principle, other non-perturbative algorithms for calculating $\hat{n}$ can be used in a similar way, for example the stroboscopic averaging method [17]. These non-perturbative algorithms can treat the synchrotron sideband resonances correctly.

The other class simulates the depolarization due to non-spin-flip synchrotron radiation using a Monte-Carlo technique to compute the depolarization rate $\lambda_{d}$, and it is a good approximation to ignore the tiny effect of kinetic polarization and estimate the equilibrium polarization in an electron(positron) storage ring following

$$
\begin{aligned}
P_{\mathrm{eq}} & \approx \frac{P_{\infty}}{1+\lambda_{d} / \lambda_{p}} \\
P_{\infty} & \approx-\frac{8}{5 \sqrt{3}} \frac{\oint \mathrm{d} \theta \frac{1}{|\rho|^{3}} \hat{b} \cdot \hat{n}_{0}}{\oint \mathrm{d} \theta \frac{1}{|\rho|^{3}}\left[1-\frac{2}{9}\left(\hat{n}_{0} \cdot \hat{s}\right)^{2}\right]}
\end{aligned}
$$

where $P_{\infty}$ is the equilibrium polarization taking into account the orbital imperfections, but disregarding the depolarization effects due to stochastic emission of synchrotron radiation. This term can be computed using a linear code like SLIM [12].

In this approach, the first-order and higher-order spin resonances are automatically handled since it is based on three-dimensional spin motion. The Monte-Carlo method does not rely on calculating $\hat{n}$ and its derivative and in any case it is valid independently of whether Eq. 3 or some other description of spin diffusion is valid. It can thus be used to check the theoretical models of the equilibrium beam polarization.

SITROS was the first code to use this pragmatic approach. It was first devel- 
oped by J. Kewisch [18, 19] and later upgraded by M. Böge [20] and M. Berglund [21]. It divided a storage ring into sections, and the break points were bending magnets where generation of "big photons" was simulated, and interaction points where weak-strong beam-beam interactions were included. Particles were transported using second-order transfer maps which included chromatic effects and the effects of sextupoles but which were not exactly symplectic. SITROS was used for HERA and LEP. D. P. Barber's SLICKTRACK [22] code also implements the "big photons" between sections, but transports particles between these break points with the thick-lens symplectic transfer matrices of SLIM. Implementation of the nonlinear orbital motion is one of its upgrade plans. Currently SLICKTRACK is well maintained and widely used, for example in the design study of electron-ion colliders and ILC damping rings.

With the great development of computing power since SITROS was first developed, it is now possible to implement such a Monte-Carlo simulation in a more "natural" way, namely with distributed generation of synchrotron-radiation photons in each integration step. Moreover, nonlinear orbital motion can be taken into account with the implementation of symplectic integrators in modern tracking codes. PTC is a tracking code developed by E. Forest [1], which was designed to model various geometries of particle accelerators, and do symplectic tracking of the orbital motion and length-preserving transport of spin [23]. Particle coordinates and Taylor maps can be tracked in a polymorphic manner, and the latter enables the normal form analysis of the one-turn map using FPP [24]. PTC is now embedded in MADX [25] and BMAD [26], and some of its functionalities can be called in MADX and BMAD as a library. On the other hand, complicated operations like implementation and correction of machine imperfections can be done using MADX or BMAD, and the lattice is then dumped to an input file for PTC. Tracking studies can be carried out inside PTC afterwards. Therefore, PTC has an ideal framework for the implementation of a Monte-Carlo simulation of the equilibrium beam polarization in electron storage rings.

The implementation of a Monte-Carlo simulation in PTC is described in 
section 1 together with its benchmarking against SODOM [16]. In section 2, we review the theory of beam polarization for ultra-high beam energies. In section 3 , a case study of the beam polarization at ultra-high beam energies is presented using this Monte-Carlo approach for a model storage ring, and compared with the theory.

\section{Code implementation and benchmarking}

When a particle (or a Taylor map) is tracked through an element in PTC, a step of integration is called an "integration node". There are five different types of integration nodes, describing the entrance patch, the entrance fringe field, the body of an element, the exit fringe field and the exit patch. Here, patches connect the local coordinates of adjacent elements. The body of an element can be split into a number of integration nodes. A schematic diagram of the integration nodes inside an element is shown in Figure .1.

In this context, synchrotron radiation effects are only taken into account for the integration nodes in the body of bending magnets. When a particle is tracked through an integration node of a magnet body, the miniscule SternGerlach effect is neglected and the orbital transfer map is sandwiched in between two spin kicks in equal amounts, and they together form a second-order symplectic integrator for the particle motion and a length-preserving rotation for the spins. The kick due to photon emission is placed next to the spin kick, and they commute with each other, as the spin-dependent synchrotron radiation is not taken into account. Therefore the synchrotron-radiation process is also concentrated before the entrance and after the exit of the orbital map in equal amounts. The transport of orbital coordinates in each integration node can be chosen from the second-order, the fourth-order and the sixth-order symplectic integrators. In this simulation study, we choose the second-order symplectic integrator. Because the quadrupoles are split into many integration nodes to ensure the accuracy of spin tracking, and dipoles are also split so that the number of photons emitted in each integration step is small, and the desired accuracy 
of orbital motion can be achieved without going to a higher-order symplectic integrator. The tracking scheme for an integration node of the body of a magnet is shown in Figure .1.

In each integration step, the local radius of curvature is computed for each tracked particle, as well as the critical energy $u_{c}$. The number of emitted photons $n_{\gamma}$ is first randomly generated with a Poisson distribution. Then the relative energy $\xi=u / u_{c}$ of each emitted photon is stochastically generated following the algorithm implemented in GEANT4 [29], which is quite fast and more precise than former implementations [28]. The stochastic energy kick before or after the orbital kick is

$$
\delta \rightarrow \delta-\sum_{1}^{n_{\gamma}} \xi u_{c} / E_{\text {beam }} .
$$

A bunch of particles are launched on the closed orbit with spins initialized along the $\hat{n}_{0}$, and are then tracked for several damping time. The beam polarization $P(t)$ as the ensemble average of these particle spins, is computed during the tracking. The depolarization rate is fitted following

$$
P(t)=\exp \left(-\frac{2 \pi c t}{C} \lambda_{d}\right)
$$

and Eq. 6 is then used to compute the equilibrium beam polarization.

The calculations with this method are benchmarked against SODOM [16] for the same model storage ring (Model 1) as described in Ref. [16]. Model 1 consists of 128 identical FODO cells and four FODO cells with vertical bends (four upward bends and four downward bends with a bending angle of 0.017453 rad, to introduce vertical dispersion). All the dipoles are 6 meters long, the quadrupoles are 1 meter long with inverse focal lengths of $k_{f}=0.18243216 \mathrm{~m}^{-1}$ and $k_{d}=0.16763610 \mathrm{~m}^{-1}$, respectively, and the cell length is 16 meters. A half ring consists of an upward-bend cell, 32 FODO cells, an RF cavity(zero length), 32 FODO cells and an upward-bend cell. In the other half ring, the upward-bend cells are replaced by downward-bend cells. The parameters are listed in Table .1. No machine imperfections are introduced in this study.

The first step is to check the evolution of the beam's eigen-emittances [30] computed using the tracking data of a beam of 9600 particles. These are then 
compared with the analytical solutions, where the beam envelope formalism [31] is used to compute the equilibrium beam emittances. As shown in Figure .2, the fitted equilibrium emittances and damping times differ from the analytical results by just a few percent.

The equilibrium polarization is calculated for the energy range of $20.71 \mathrm{GeV}$ to $20.84 \mathrm{GeV}$, where several synchrotron-sideband spin resonances are visible. The expectation number of the emitted photons is $\sim 11$ for the main dipole. Therefore, the number of integration steps is chosen to be 10 and it is unlikely that a large number of photons are generated at each integration node. It takes around a minute to track a particle for 3000 turns (5 damping times) using CPUs of the Hopper cluster at the National Energy Research Scientific Computing Center(NERSC), and the tracking of different particles can be parallelized. Several simulations with the same number of particles can be launched. The depolarization rate is the average of the results of these simulations, and the statistical error can also be calculated. A comparison of the Monte-Carlo result with 50 particles and SODOM is shown in Figure .3. The results are consistent with each other.

There are several possible issues for this Monte-Carlo simulation. First, if the depolarization rate is very low then it would take an impractically long time to reach an accurate estimation of the depolarization rate and hence the equilibrium beam polarization. Second, since the particles are launched on the closed orbit, it takes many turns for particles to diffuse to large amplitudes, and the contribution from phase space with large amplitudes is likely to be underestimated. To solve this problem, the beam can be given a Gaussian distribution at the start [19]. Note that the particle spins should then be initialized to be parallel to the ISF. In addition, it is arguable whether implementation of nonlinear orbit motion and photon emission at each integration step are necessary for all practical simulations. However, it is not trivial to turn off the nonlinear orbit motion in the tracking. Then the linear transfer matrices obtained by tracking the first-order Taylor map could be used instead and the algorithm of SLICKTRACK then realized with some effort. Moreover, the number of integration 
steps of bending magnets can be easily set in PTC, and computing time can be reduced when needed.

\section{Further details of the theory}

Electron(positron) polarization has been observed and studied in several storage rings $[32,33]$ and the polarizations attained have been broadly in line with the expectations of Eq. 3. In storage rings with relatively low beam energies, the first-order spin resonances of Eq. 2 dominate together with their synchrotron sidebands. Resonances can have several origins including transverse coupling, lack of so-called spin transparency in regions where $\hat{n}_{0}$ is horizontal due to the use of spin rotators, and indirect effects resulting from orbital imperfections. Various spin matching schemes [11] have been studied and implemented in several machines to weaken the first-order resonances and these schemes bring improvements in the achievable equilibrium polarization. In high energy storage rings, it is generally believed that the synchrotron sideband resonances are the most important family of spin resonances.

We have already described how synchrotron sideband resonances have their origin in the modulation of the spin precession rate due to synchrotron motion. We now look in more detail.

As mentioned earlier, near spin-orbit resonance the ISF, $\hat{n}$, can be strongly spread out away from $\hat{n}_{0}$. Then, even in the absence of radiation, the maximum attainable equilibrium polarization of the beam, $\langle\hat{n}\rangle$, can be be small. In particular, in the case of synchrotron sideband resonances, $\hat{n}$ can vary strongly as a function of the synchrotron phase with the same orbital actions and this can contribute to making the spin-orbit coupling function large. Then the radiative depolarization can be strong and lead to small equilibrium beam polarization according to Eq. 3. Here, we have used a standard picture in which we treat the betatron and synchrotron motion on an equal footing. However, we can gain extra insight by treating the betatron and synchrotron motion separately and by noting that if the modulation of the spin precession rate causes an initially 
vertical spin to cross a parent betatron spin resonance, the spin can be strongly disturbed and might even flip over as expected from the Froissart-Stora formula [34] and as illustrated in [35, pp.74-75]. Although this argument is heuristic in that it likens the modulation of the spin precession frequency to a modulation of the closed orbit spin tune $\nu_{0}$, it contains the essentials. Note that in this description the typical rate of resonance crossing increases with $\nu_{z}$. Both of these pictures assume long-term coherence or near coherence between relevant terms in $\vec{\omega}$ and the basic spin motion.

Note that the introduction of Siberian snakes $[36,37]$ might reduce the dependence of spin transport on the energy oscillations of synchrotron motion so that that mechanism could be suppressed [39]. This topic is beyond the scope of this paper.

The synchrotron sideband resonances of an integer resonance are $\nu_{0}=k+$ $m \nu_{z}$, with the contribution to $\lambda_{d}^{0} / \tilde{\lambda}_{p}[40]$

$$
\frac{\lambda_{d}^{0}}{\tilde{\lambda}_{p}}=A \sum_{m=-\infty}^{\infty}\left[\frac{\Delta \nu}{\left(\Delta \nu+m \nu_{z}\right)^{2}-\nu_{z}^{2}}\right]^{2} e^{-\alpha} I_{m}(\alpha)
$$

where $\Delta \nu$ is the distance between the closed orbit spin tune and the parent resonance, say $\Delta \nu=\nu_{0}-k,|m|$ is the order of the sideband resonance, $I_{m}$ is a modified Bessel function, $\sigma_{\epsilon}$ is the rms relative energy spread, and $\alpha=$ $\left(\nu_{0} \sigma_{\epsilon} / \nu_{z}\right)^{2}$ the tune modulation index. $A$ is a constant that relates to the width of the first-order synchrotron sideband resonance, because if we set $\alpha=0$, then

$$
\left.\frac{\lambda_{d}^{0}}{\tilde{\lambda}_{p}}\right|_{\alpha=0}=\frac{A \Delta \nu^{2}}{\left(\Delta \nu^{2}-\nu_{z}^{2}\right)^{2}} .
$$

The synchrotron sideband resonances of an isolated horizontal parent resonance are $\nu_{0}=k \pm \nu_{x}+m \nu_{z}$, with the contribution to $\lambda_{d}^{0} / \tilde{\lambda}_{p}[40]$

$$
\begin{aligned}
\frac{\lambda_{d}^{0}}{\tilde{\lambda}_{p}}=B \sum_{m=-\infty}^{\infty} & \left\{\frac{e^{-\alpha}}{\left(\Delta \nu+m \nu_{z}\right)^{2}} \times\right. \\
& {\left[J_{x} I_{m}(\alpha)+J_{z} \frac{\alpha}{2}\left(I_{m-1}(\alpha)+I_{m+1}(\alpha)\right)\right]+} \\
& \left.\frac{e^{-\alpha}}{\left(\Delta \nu+m \nu_{z}\right)} \frac{m J_{z}}{\nu_{z}} I_{m}(\alpha)\right\}
\end{aligned}
$$


where $\Delta \nu=\nu_{0}-\left(k \pm \nu_{x}\right), J_{x}$ and $J_{z}$ are the horizontal and longitudinal damping partition numbers and $B$ is a constant that relates to the width of the horizontal parent resonance. If we set $\alpha=0$, then

$$
\left.\frac{\lambda_{d}^{0}}{\tilde{\lambda}_{p}}\right|_{\alpha=0}=\frac{B J_{x}}{\Delta \nu^{2}} .
$$

The result for synchrotron sidebands for an isolated vertical parent resonance can be obtained by replacing $x$ by $y$ in Eq. 12. The equilibrium polarization can then be estimated as

$$
P_{\mathrm{eq}} \approx \frac{P_{0}}{1+\sum_{\nu_{k}}\left(\frac{\lambda_{d}^{0}}{\tilde{\lambda}_{p}}\right)_{\nu_{k}}},
$$

where $\nu_{k}=k_{0}+k_{x} \nu_{x}+k_{y} \nu_{y}+k_{z} \nu_{z}$ is the location of a parent resonance. Therefore, once we have computed the equilibrium polarization using a linear formalism like SLIM, the widths $A$ and $B$ of the first-order (betatron and synchrotron) spin resonances can be fitted and the equilibrium polarization taking into account the synchrotron sideband resonances can then be estimated analytically using Eq. 13. Note, however, that the nearby first-order horizontal and vertical parent spin resonances might not be well separated and that resulting interference effects are not included in Eq. 13.

Note that these calculations of the strengths of the synchrotron sideband resonances are based on evaluation of the spin-orbit coupling function in Eq. 3 and that that assumes that the synchrotron motion is well defined so that the synchrotron phases are strongly correlated from turn to turn. However, it has been suggested that for ultra-high beam energies, it is possible to enter a new regime whereby the successive passages of spin resonances during synchrotron oscillations are uncorrelated [2] and that this can modify the rate of depolarization.

In fact in Ref. [41], it was argued that Eq. 3 should be generalized when the system is very close to a spin resonance. In particular, following the heuristic physical explanation in Ref. [42], very close to a spin resonance, stochastic 
photon emissions should contribute a depolarization rate of

$$
\lambda_{d}^{\prime}=\pi \sum_{k}\left\langle\left|\omega_{k}\right|^{2} \delta\left(\nu_{s}-\nu_{k}\right)\right\rangle
$$

So an additional term was added to $\alpha_{+}$in Eq. 3 when evaluating the equilibrium beam polarization to give

$$
\begin{aligned}
\alpha_{+}= & \oint \mathrm{d} \theta\left\langle\frac{1}{|\rho|^{3}}\left[1-\frac{2}{9}(\hat{n} \cdot \hat{\beta})^{2}+\frac{11}{18}\left(\frac{\partial \hat{n}}{\partial \delta}\right)^{2}\right]\right\rangle+ \\
& \frac{8}{5 \sqrt{3}} \frac{2 \pi c}{C} \frac{m_{e}}{r_{e} \gamma^{5} \hbar} \pi \sum_{k}\left\langle\left|\omega_{k}\right|^{2} \delta\left(\nu-\nu_{k}\right)\right\rangle .
\end{aligned}
$$

where the perturbed spin precessing frequency is $\nu=\nu_{0}+\Delta \nu$ with $\Delta \nu$ due to the energy oscillation, $\nu_{k}=k+k_{x} \nu_{x}+k_{y} \nu_{y}+k_{z} \nu_{z}$ is the location of a spin resonance, and $\omega_{k}$ is the amplitude of the Fourier component for the first-order spin resonances. The average in the additional term is taken over the beam distribution.

In summary, it was suggested that the total depolarization rate should be $\lambda_{d}=\lambda_{d}^{0}+\lambda_{d}^{\prime}$. The additional term should not be important if the spread of the spin precession frequency is small, because we do not run a machine very close to a major spin resonance.

Now let us estimate the spread in the spin phases with synchrotron oscillations and stochastic photon emission. Following the derivation in Ref. [43], the variation of the spin precession phase $\Phi$ can be expressed as

$$
\frac{d \Phi}{d \theta}=a \gamma=a \gamma_{0}(1+\delta),
$$

Let $\Delta \delta_{i}$ be the change of $\delta$ due to the emission of a photon at $\theta=\theta_{i}$. Then the spin phase will be shifted by the amount

$$
a \gamma_{0} \Delta \delta_{i} \int_{\theta_{i}}^{\theta^{\prime}} \cos \nu_{z}\left(\theta^{\prime}-\theta_{i}\right) d \theta^{\prime}=\frac{\nu_{0}}{\nu_{z}} \Delta \delta_{i} \sin \nu_{z}\left(\theta-\theta_{i}\right),
$$

at the azimuthal angle $\theta$ after the emission. Then summing up all the effects of photon emissions between $\theta=0$ and $\theta=\Theta$, the total variation of spin phase is

$$
\Delta \Phi(\theta)=\frac{\nu_{0}}{\nu_{z}} \sum_{0<\theta_{i}<\Theta} \Delta \delta_{i} \sin \nu_{z}\left(\Theta-\theta_{i}\right)
$$


Averaging $(\Delta \Phi(\theta))^{2}$ on time over $(0, \Theta)$ and on all the emissions that occur during this period of time,

$$
\left\langle(\Delta \Phi(\theta))^{2}\right\rangle=\left(\frac{\nu_{0}}{\nu_{z}}\right)^{2}\left\langle\left(\Delta \delta_{i}\right)^{2}\right\rangle \frac{d N}{d \theta} \int_{0}^{\Theta} d \theta^{\prime} \sin ^{2} \nu_{z}\left(\Theta-\theta^{\prime}\right) .
$$

Here $d N / d \theta$ is the mean number of emitted photons during one radian of $\theta$. Since [44]

$$
\frac{d N}{d \theta}\left\langle\left(\Delta \delta_{i}\right)^{2}\right\rangle=\frac{11}{9} \tilde{\lambda}_{p}
$$

we then get the spread in spin phase in a synchrotron oscillation period

$$
\left\langle(\Delta \Phi)^{2}\right\rangle=\frac{11}{18}\left(\frac{\nu_{0}^{2}}{\nu_{z}^{3}}\right) \tilde{\lambda}_{p}
$$

We define the correlation index as $\kappa=\frac{11}{18} \nu_{0}^{2} \tilde{\lambda}_{p} / \nu_{z}^{3}$. If $\kappa \ll 1$, the spread of spin phase is small, then the successive passages of the spin resonance due to synchrotron oscillation are correlated.

Otherwise, if the rms spread of the spin-precession frequency $\sigma_{\nu}=\nu_{0} \sigma_{\delta} \gg \nu_{z}$, the crossings of resonances during synchrotron motion are completely uncorrelated. It is claimed that in this regime the synchrotron oscillation plays the role of SR photon emission in driving the uncorrelated resonance crossings, and that the depolarization rate is described by Eq. 14. Moreover, the spin resonances completely overlap and are unavoidable. Then it is not clear to us whether the analytical calculation of Eq. 3 for $\lambda_{d}^{0}$ is still applicable and, if it is not applicable, it is not clear to us what prescription would be used instead. Furthermore it is not clear to us whether the total depolarization rate can be obtained by simply adding $\lambda_{d}^{0}$ and $\lambda_{d}^{\prime}$ together.

In Ref. [2], it was suggested that when $\sigma_{\nu} \ll 1, \lambda_{d}^{\prime}$ and $\lambda_{d}^{0}$ would be comparable, and should be added together to obtain the depolarization rate $\lambda_{d}$. Moreover, in Ref. [2] only the contributions from the parent resonances were used to calculate $\lambda_{d}^{0}$. When $\sigma_{\nu} \gg 1, \lambda_{d}^{0}$ is negligibly small and it was predicted there there would be no resonant dependence of spin diffusion on energy, and that beam polarization would increase with energy.

The above idea was applied to the LEP storage ring [45] and it was shown that the depolarization should have entered the uncorrelated regime for LEP 
above $60 \mathrm{GeV}$. A new optics was implemented in LEP which facilitated establishing polarization at $60.6 \mathrm{GeV}$ [46], whereas a beam polarization of below $1 \%$ was measured at $70 \mathrm{GeV}, 92 \mathrm{GeV}$ and $98.5 \mathrm{GeV}$, in qualitative agreement with Eq. 3. So the hypothetical increase of polarization at high energy was not observed.

\section{Simulation study of a model storage ring}

For this study we adopted a simplified version of the CEPC lattice (Model 2). It has a periodicity of $P=4$, with one superperiod shown in Figure .4 . The arc sections are composed of FODO cells with $60^{\circ}$ phase advance in both transverse planes and they have dispersion suppressors with missing dipoles. The simplified straight sections are composed of FODO cells without taking the injection insertions into account. Identical RF cavities of zero length are placed in the center of each straight section. The " $2 \pi$ phase advance" section occupies the space left for the final focus insertion. Four identical thin skew quadrupoles are inserted at the center of each superperiod and the transverse emittance ratio is tuned to 0.003. No orbit imperfections are added in the simulations, and the first-order synchrotron sideband resonances are generally weak. For a four-fold symmetric ring the parent betatron spin resonances are in principal located at

$$
\nu_{0}=4 k \pm \nu_{j}, \quad j=x, y \text { and } k \in \mathbb{Z}
$$

In this section, two cases of different energy ranges will be studied around $120 \mathrm{GeV}$ and $150 \mathrm{GeV}$, respectively. The parameter list relevant for this study is shown in Table .2, Note that for these parameters we take into account the effect of the sawtooth shape of the closed caused by synchrotron radiation in the dispersive regions of the ring.

The equilibrium polarization has been computed in three different ways which are compared with each other. First, the Monte-Carlo approach described in section 2 has been used to calculate the equilibrium polarization for $a \gamma_{0}$ between 267.5 and 275.5 for Case 1, and between 338.5 and 346.5 for Case 2, 
the step size for both cases being $a \gamma_{0}=0.01$. The expected number of emitted photons in a main dipole is $\sim 8$ for $120 \mathrm{GeV}$ and $\sim 10$ for $150 \mathrm{GeV}$, therefore, the number of integration steps is chosen to be 10 and it is unlikely that a large number of photons are generated at each integration node. Sixty particles are tracked for each energy point, and each particle is tracked for 10 damping times, which takes around 4 minutes for $120 \mathrm{GeV}$ using the CPUs of the Hopper cluster at NERSC.

Second, a first order Taylor map is tracked for one turn and $\partial \vec{n} / \partial \delta$ is computed with a normal form [14]. Then the equilibrium polarization is calculated using Eq. 3 with only the first-order spin resonances. Following the theory of synchrotron sideband resonances in the correlated regime, the constants $A$ or $B$ for each first-order spin resonance are fitted using Eq. 10 and Eq. 12. Since the widths of some first-order spin resonances are very small, and thus their synchrotron sidebands are even narrower, these resonances and their synchrotron sidebands contribute to the equilibrium beam polarization as very narrow dips. Since the step size of the Monte-Carlo simulation is $a \gamma_{0}=0.01$, some of these very narrow dips are beyond the resolution of the Monte-Carlo simulation, and thus they are irrelevant for our comparison between different methods. As shown in Fig .5, only the resonances with $A(B)>10^{-5}$ are retained in the fitting, and no first-order synchrotron spin resonance qualifies for Case 1 and Case 2. Note that for Case 1 near $a \gamma_{0}=269$, and for Case 2 near $a \gamma_{0}=341$, the two nearby first-order spin resonances overlap with each other, so that there is some discrepancy between the fitted curve and the simulation result. Then the equilibrium polarization taking into account of the synchrotron sidebands is calculated following Eq. 11 and Eq. 13 including only the first-order betatron spin resonances with $B>10^{-5}$.

Third, following the theory of spin diffusion in the uncorrelated regime, the strengths of the first-order resonances $\nu_{0}=k \pm \nu_{x}$ and $\nu_{0}=k \pm \nu_{y}$ are computed with PTC using a normal form [14]. For each of these first-order resonances, the strength scales with the square root of the particle's action, and when applying Eq. 14 to calculate the depolarization rate $\lambda_{d}^{\prime}$, an average is 
taken over the equilibrium beam distribution. Assuming that the beam has a Gaussian distribution, Eq. 14 becomes

$$
\frac{\lambda_{d}^{\prime}}{\tilde{\lambda}_{p}}=\sum_{k} \frac{2 \sqrt{\pi}\left|\omega_{k 0}\right|^{2}}{\sigma_{\nu} \tilde{\lambda}_{p}} e^{-\left(\nu_{0}-\nu_{k}\right)^{2} / \sigma_{\nu}^{2}},
$$

where $\omega_{k 0}$ is the strength of resonance $\nu_{0}=\nu_{k}$ of a particle whose actions correspond to the equilibrium emittances. It is easy to see from the known dependences of $\tilde{\lambda}_{p}, \omega_{k 0}$ and $\sigma_{\nu}$ on the beam energy that $\lambda_{d}^{\prime} / \tilde{\lambda}_{p}$ decreases strongly as the beam energy increases. The $\omega_{k 0}$ of the first-order resonances in the two energy ranges are shown in Figure .6. Since it is not clear to us whether $\lambda_{d}^{0}$ should be added to the depolarization rate as well, we just ignore its contribution in this method.

Results for the three methods are shown in Figure .7 for both cases. For Case 1 , the correlation index is 0.174 , much smaller than 1 , and the Monte-Carlo result is more consistent with the expectation for correlated regime, where fine resonance structures are observed. For Case 2, the correlation index is 1.160, and according to the criterion, it is regarded as being in the uncorrelated regime. The Monte-Carlo result does not show clear synchrotron-sideband structures, and it looks more consistent with that of the uncorrelated regime. The discrepancy between the result of the Monte-Carlo simulation and that for the "uncorrelated regime" might be due to the ignored contribution from $\lambda_{d}^{0}$. This needs further verification and that requires a code to evaluate the spin orbit coupling function non-perturbatively. SODOM and stroboscopic averaging are two possible options, but their application in such ultra-high beam energies also needs justification. This is beyond the scope of this paper.

In any case, so far, and without misalignments, the results of the simulations support the theory of the uncorrelated resonance crossing in synchrotron oscillations. In addition, it also indicates Eq. 3 is not applicable for evaluating the equilibrium beam polarization in the uncorrelated regime. 


\section{Conclusion}

This paper presents Monte-Carlo simulations of the equilibrium beam polarization in electron storage rings, on the basis of the PTC code, which treats nonlinear orbit and three-dimensional spin motion, and which generates synchrotronradiation photons at each integration step. The simulations are benchmarked against SODOM [16], and limitations are also discussed. Monte-Carlo simulations are launched to study a model storage ring with parameters similar to those of the proposed CEPC, and the results are consistent with the suggestions for the correlated and uncorrelated regimes of beam depolarization at ultra-high beam energies.

In particular, the results are consistent with the notion that already at $150 \mathrm{GeV}$ more polarization is attainable than that predicted by Eq. 3 with the analytical solution for synchrotron sideband resonances. This is obviously very important. Nevertheless, we feel that the notion of uncorrelated resonance crossing should be put on a firmer mathematical basis. A good starting point would be rigorous solution of the evolution equation for the polarization density [47]. In any case, the simulations suggest that use of a Monte-Carlo method is the most effective way to proceed at present. As far as we are aware this is the first serious numerical study of this kind for ultra-high beam energies.

At this stage of our studies our models do not have misalignments and the resulting distortions of the closed orbit. However, the simulation of realistic misalignments and the modeling of the correction of the orbital imperfections is very important for beam polarization at ultra-high beam energies. These topics will be studied in the future on the basis of this simulation framework, and will be an integral part of investigation of feasibility of attaining high beam polarization at CEPC. Nevertheless our current results are not just of academic interest.

For the attainment of polarized beam at not-so-ultra-high beam energies (for example $120 \mathrm{GeV}$ ), it appears to be necessary to find some way to reduce the spread in the spin-precession rate. It has been suggested that this could be 
achieved by manipulating the dispersion functions [48] or by reducing the energy spread with the aid of special nonlinear wigglers $[49,50]$. However, it is far from clear that such schemes are practical. An alternative would be to introduce two Siberian snakes. For this, $\hat{n}_{0}$ points upwards in one half ring and it points downwards in the other half so that Sokolov-Ternov effect is suppressed. This could be overcome by having short strong dipoles in one half ring and weak long dipoles in the other half. A simulation on this at lower energies [39] has already shown a suppression of synchrotron sideband resonances.

This work is supported by the Hundred-Talent Program (Chinese Academy of Sciences), and National Natural Science Foundation of China (11105164). We would like to thank Drs. D. Abell and E. Forest on their help with the simulation code PTC. One of us, Z. Duan, would like to thank Drs. S. Mane, U. Wienands and K. Yokoya for helpful discussions in theoretical aspects. The simulation work used the resources of the National Energy Research Scientific Computing Center, a DOE Office of Science User Facility supported by the Office of Science of the U.S. Department of Energy under Contract No. DE-AC02-05CH11231.

[1] F. Schmidt, E. Forest, E. McIntosh, CERN-SL-2002-044-AP, KEKREPORT-2002-3 (2002).

[2] Y. Derbenev, A. Kondratenko, A. Skrinsky, Part. Accel. 9 (1979) 247.

[3] CEPC, http://cepc.ihep.ac.cn.

[4] FCC-ee, http://tlep.web.cern.ch.

[5] L. H.Thomas, Phil.Mag. 3 (1927) 1-21.

[6] V. Bargmann, L. Michel, V. L. Telegdi, Phys. Rev. Lett. 2 (1959) 435.

[7] D. P. Barber, J. Ellison, K. Heinemann, Phys.Rev. ST Accel.Beams 7 (2004) 124002.

[8] A. Sokolov, I. Ternov, Sov. Phys. Doklady 8 (1964) 1203.

[9] V. N. Baier, Y. Orlov, Sov. Phys. Doklady 10 (1966) 1145. 
[10] Y. Derbenev, A. Kondratenko, Sov. Phys. JETP 37 (1973) 968-973.

[11] D. P. Barber, G. Ripken, Handbook of accelerator physics and engineering, edited by A. W. Chao and M. Tigner, 1st edition, 3rd printing, World Scientific (2006) and 2nd edition, edited by A.W. Chao, K.H. Mess, M. Tigner and F. Zimmermann (2013).

[12] A. Chao, Nucl. Instrum. Meth. 180 (1981) 29.

[13] S. Mane, Phys. Rev. A 36 (1987) 120.

[14] D. .T. Abell, D. Meiser, D. P. Barber and E. Forest, talk on ICAP 2012, Rostock-Warnemnde, Germany, 2012.

[15] S. Mane, Private communication.

[16] K. Yokoya, KEK Report KEK-92-6, KEK (1992).

[17] K. Heinemann, G. H. Hoffstätter, Physical Review E 54 (1996) 4240.

[18] J. Kewisch, DESY Report DESY-83-032, DESY (1983).

[19] J. Kewisch, R. Rossmanith, T. Limberg, Phys. Rev. Lett. 62 (1989) 419.

[20] M. Boge, DESY report, DESY-94-087, DESY (1994).

[21] M. Berglund, Doctoral thesis, Royal Institute of Technology, Stockholm, Sweden (2001) and Report DESY-Thesis-2001-044 (2001).

[22] D. P. Barber, in: Proc. 16th Int. Spin Physics Symp., World Scientific, Kyoto, Japan, 2005.

[23] S. Mane, KEK Report KEK-2009-8, KEK (2009).

[24] E. Forest, Y. Nogiwa, in: Proc. ICAP 2006, Chamonix, France, 2006.

[25] Methodical Accelerator Design (MAD) program, CERN, http://cern.ch/mad/. 
[26] D. Sagan, Nucl. Instrum. Meth. A 558 (2006) 356.

[27] Y. Cai, SLAC-PUB-7793, SLAC (1998).

[28] G. Roy, Nucl. Instrum. Meth. A 298 (1990) 128.

[29] H. Burkhardt, EUROTEV-REPORT-2007-018, CLIC-NOTE-709, CERNOPEN-2007-018, CERN (2007).

[30] Y. Alexahin, arXiv:1409.5483 (2014).

[31] K. Ohmi, K. Hirata, K. Oide, Phys. Rev. E. 49 (1994) 751.

[32] S. Mane, Y. M. Shatunov, K. Yokoya, Rep. Prog. Phys. 68 (2005) 1997.

[33] D. Barber, et al., Phys. Lett. B343 (1995) 436.

[34] M. Froissart and R. Stora, Nucl. Instrum. Meth. 7 (1960) 297.

[35] G. H. Hoffstaetter, "High-energy polarized proton beams: A modern view. Springer Tracts in Modern Physics", Volumn 218, 2006.

[36] Y. Derbenev, A. Kondratenko, in: Proc. 10th Int. Conf. on High Energy Accelerators, Protvino, USSR, 1977.

[37] Y. Derbenev, A. Kondratenko, Part. Accel. 8 (1978) 115.

[38] D. Abell, PTC Library User Guide, Tech-X Corporation, unpublished, 2011.

[39] D.P. Barber, H-U. Wienands, M. Fitterer and H. Burkhardt, in: Proc. 19th Int. Spin Physics Symposium, Juelich, Germany, 2010.

[40] S. Mane, Nucl. Instrum. Meth. A292 (1990) 52.

[41] Y. Derbenev, A. Kondratenko, Sov. Phys. Doklady 19 (1975) 438.

[42] A. Kondratenko, Sov. Phys. Doklady 39 (1974) 592.

[43] K. Yokoya, Part. Accel. 14 (1983) 39. 
[44] M. Sands, SLAC-R-121, SLAC (1970).

[45] R. Assmann, in: Proc. PAC 1999, New York City, USA, 1999.

[46] R. Assmann, et al., CERN-SL-2000-012, CERN (2000).

[47] K. Heinemann and D.P. Barber, Nucl. Instr. Meth. A 463 (2001) 62 and 469 (2001) 294.

[48] K. Yokoya, Part. Accel. 13 (1983) 95.

[49] A. Hofmann and J. M. Jowett, CERN/ISF-TH/81-23, CERN (1981).

[50] J. M. Jowett, CERN/ISF-TH/81-24, CERN (1981).

Table .1: The parameters of Model 1 storage ring

\begin{tabular}{cc}
\hline Parameter & Value \\
\hline Circumference $(\mathrm{m})$ & 2112 \\
Beam energy $(\mathrm{GeV})$ & 20.736 \\
$\nu_{x} / \nu_{y} / \nu_{z}$ & $33.265 / 28.380 / 0.0623$ \\
Relative energy spread & $1.13 \times 10^{-3}$ \\
damping time $($ turns $)$ & $620 / 620 / 310$ \\
emittance $(\operatorname{mm} \cdot \operatorname{mrad})$ & $8.03 \times 10^{-2} / 2.38 \times 10^{-4} / 7.42$ \\
Sokolov-Ternov time $\left(\tau_{p}\right)(\operatorname{minute})$ & 0.053 \\
rms spin precession frequency spread $\left(\sigma_{\nu}\right)$ & 0.732 \\
modulation index $(\alpha)$ & 0.012 \\
correlation index $(\kappa)$ & \\
\hline
\end{tabular}


Table .2: The parameters of Model 2 storage ring

\begin{tabular}{ccc}
\hline Parameter & Case 1 & Case 2 \\
\hline Circumference $(\mathrm{m})$ & 54752 & 54752 \\
Beam energy $(\mathrm{GeV})$ & 120 & 150 \\
$\nu_{x} / \nu_{y} / \nu_{z}$ & $193.084 / 193.218 / 0.181$ & $193.088 / 193.216 / 0.162$ \\
Relative energy spread & $1.3 \times 10^{-3}$ & $1.64 \times 10^{-3}$ \\
damping time(turns) & $80 / 80 / 41$ & $41 / 41 / 21$ \\
emittance $(\operatorname{mm} \cdot \operatorname{mrad})$ & $2.26 \times 10^{-3} / 1.92 \times 10^{-5} / 2.8$ & $9.78 \times 10^{-3} / 2.92 \times 10^{-5} / 4.9$ \\
Sokolov-Ternov time $\left(\tau_{p}\right)(\operatorname{minute})$ & 0.358 & 7.0 \\
rms spin precession frequency spread $\left(\sigma_{\nu}\right)$ & 3.921 & 0.560 \\
modulation index $(\alpha)$ & 0.174 & 11.986 \\
correlation index $(\kappa)$ & & 1.160 \\
\hline
\end{tabular}




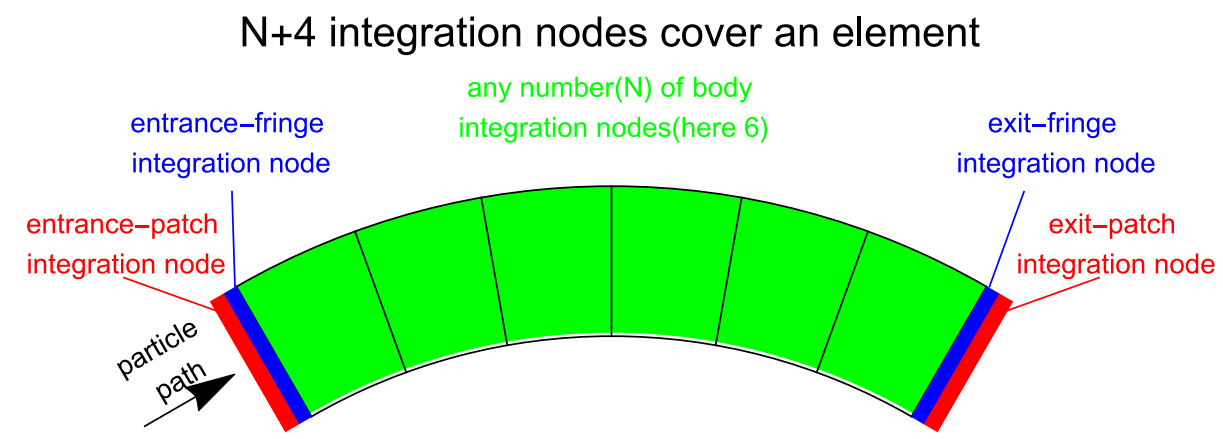

The symplectic integrator of a body integration node

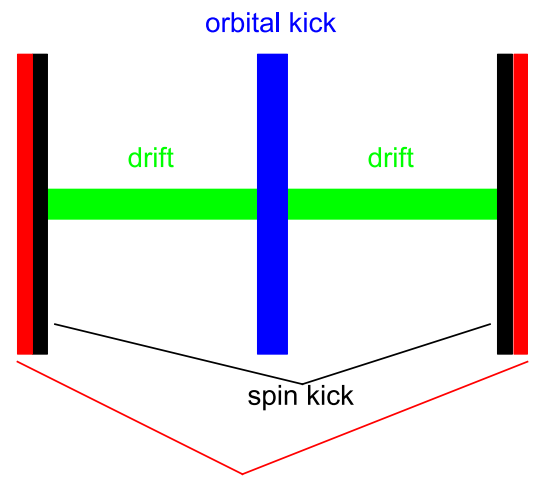

photon-emission energy kick

Figure .1: A schematic diagram showing the tracking scheme in PTC. The top part shows the integration nodes inside an element [38], while the bottom part shows the scheme of symplectic integration in a body integration node, and photon emission. 


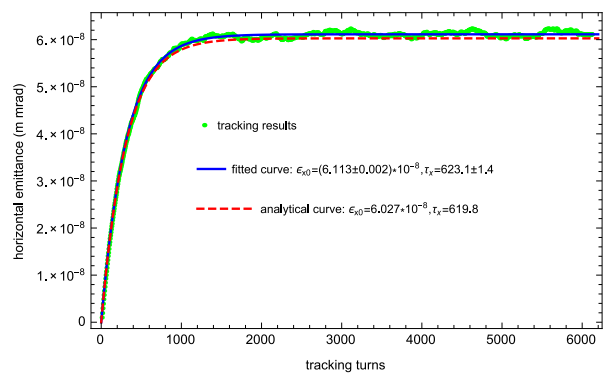

(a)

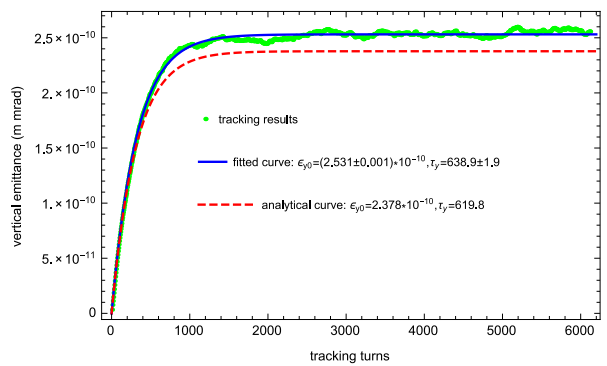

(b)

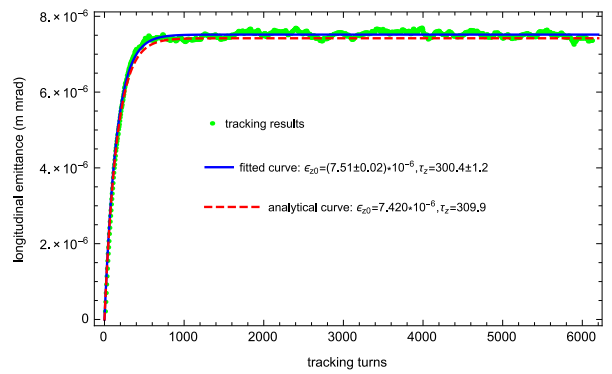

(c)

Figure .2: Evolution of eigen-emittances of a beam of 9600 particles in the Monte-Carlo simulation for Model 1. Plots (a), (b) and (c) show the horizontal/vertical/longitudinal beam eigen-emittances, respectively. The evolutions of the emittances are fitted following $\epsilon_{i}(t)=$ $\epsilon_{i 0}\left(1-\exp \left(-t / \tau_{i}\right)\right), i=x, y, z$. Comparison of the fitted parameters and the analytical solutions are also shown in the figures. 


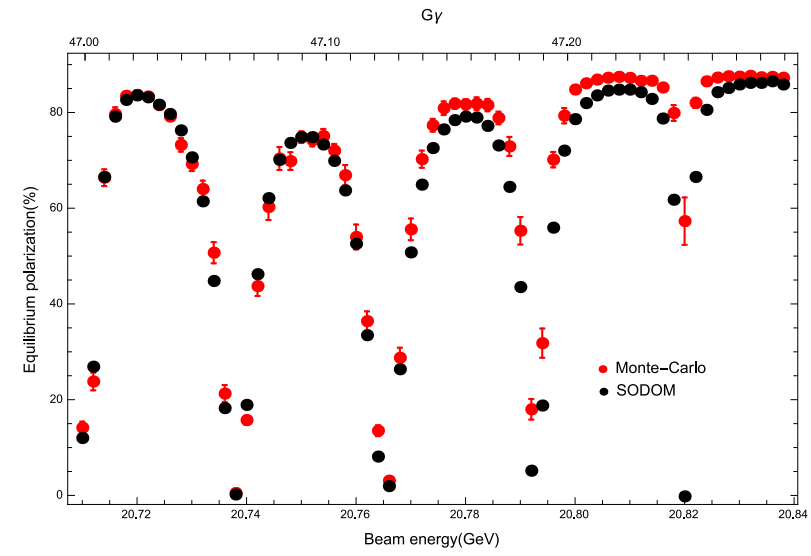

Figure .3: Comparison of the computed equilibrium polarizations for Model 1. "SODOM" is taken from the Yokoya's paper [16] with his permission. "Monte-Carlo" is the Monte-Carlo simulation result with 50 particles, the statistical error is calculated with 20 such simulations. The agreement is good.

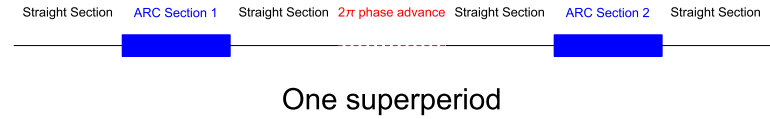

Figure .4: A schematic plot of a superperiod of the Model-2 storage ring. ARC sections 1 and 2 are identical. Therefore two identical Straight-Arc-Straight sections are connected with a section with $2 \pi$ phase advance. 


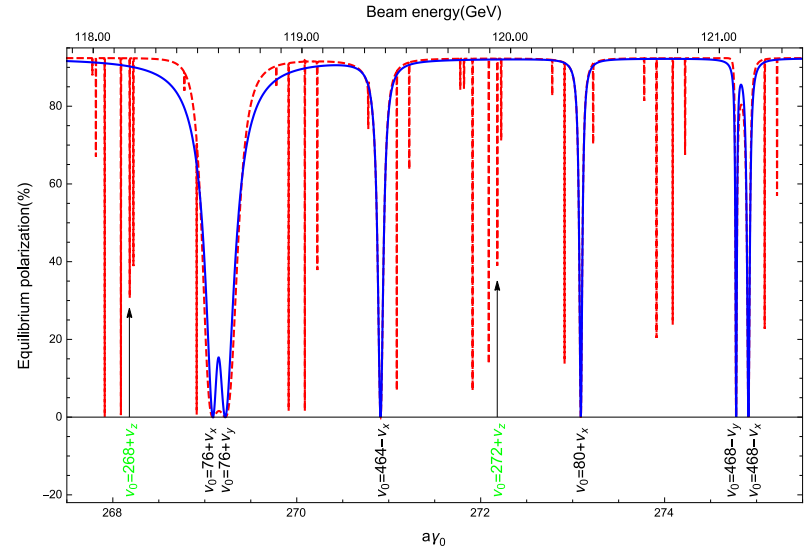

(a) Case 1

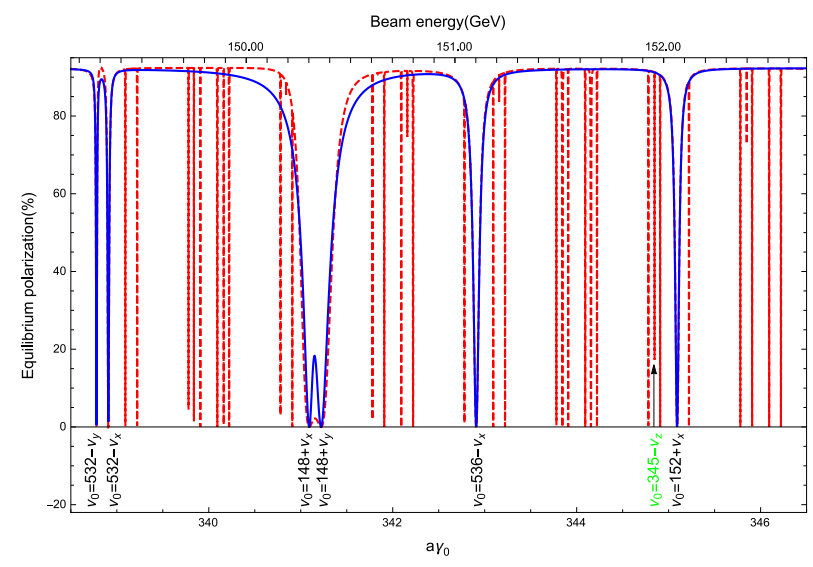

(b) Case 2

Figure .5: Scan of the equilibrium polarization versus beam energy (closed orbit spin tune) with only first-order spin resonances. The dashed line shows the equilibrium polarization computed with a normal form in PTC and the solid line shows the curve fitted using Eq. 12. Here, only the betatron spin resonances with $B>10^{-5}$ are retained in the fitting and their locations are indicated at the bottom of the plots. Note that there is no first-order synchrotron spin resonance with $A>10^{-5}$. The locations of some first-order synchrotron spin resonances are also marked in the plots in green and with arrows. 


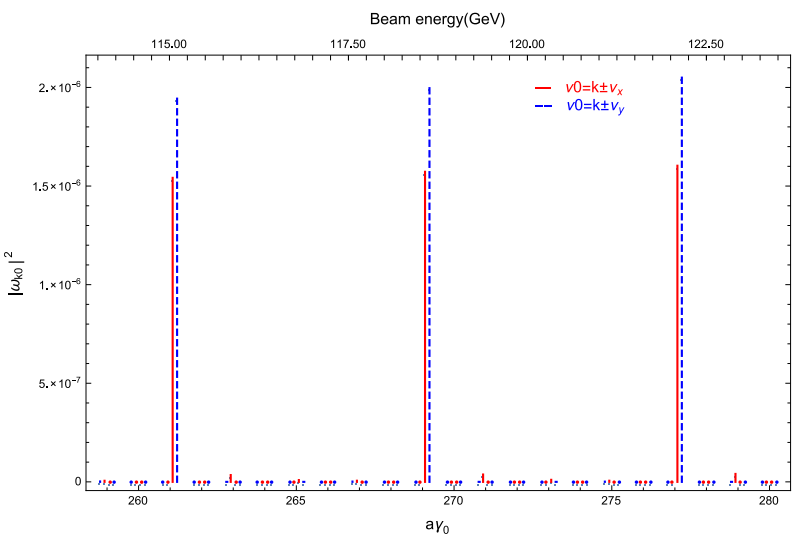

(a) Case 1

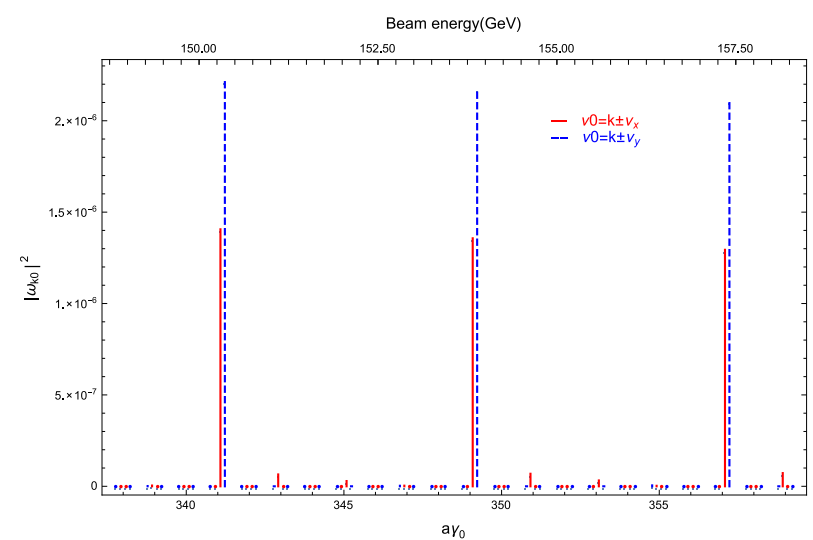

(b) Case 2

Figure .6: The spectrum of the first-order resonance strengths computed with a normal form in PTC. The resonance strengths are for particle actions corresponding to the equilibrium beam emittances. The solid and dashed lines represent the horizontal and vertical spin resonances, respectively. 


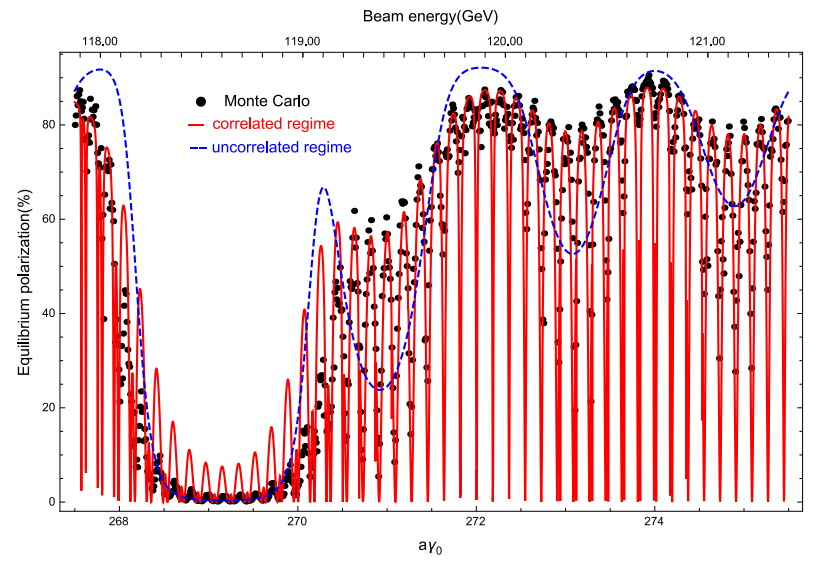

(a) Case 1

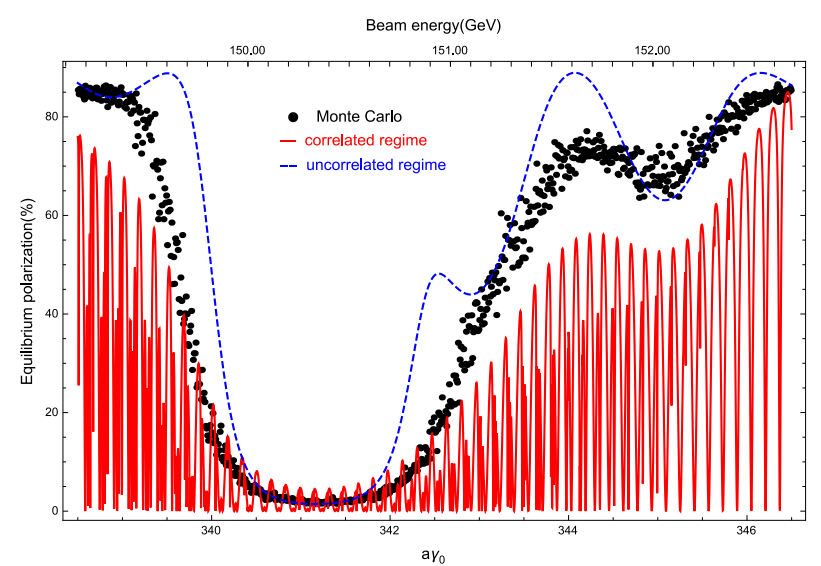

(b) Case 2

Figure .7: Comparison of the equilibrium polarizations computed by the three different methods. The first method is the Monte-Carlo simulation. The second method first fits the widths of parent spin resonances using Eq. 12, and then computes the synchrotron sideband resonances following Eq. 11, and it is labelled as "correlated regime" in this figure. The third method, referred to as "uncorrelated regime" in this figure, computes the strengths of nearby first-order spin resonances using a normal form, and then applies Eq. 23. 